\title{
DEVELOPMENT OF DATA ACQUISITION ROBOT USING WSN - KROTO FINDER
}

\author{
Arun Kumar S K \\ Department of Electronics and Communication \\ Nitte Meenakshi Institute of Technology, Bangalore, \\ Karnataka, India \\ Chaithanya Borker Y \\ Department of Electronics and Communication \\ Nitte Meenakshi Institute of Technology, Bangalore, \\ Karnataka, India
}

\author{
Ashish S Shetty \\ Department of Electronics and Communication \\ Nitte Meenakshi Institute of Technology, Bangalore, \\ Karnataka, India \\ Harish Solpure \\ Department of Electronics and Communication \\ Nitte Meenakshi Institute of Technology, Bangalore, \\ Karnataka, India
}

\author{
Dr. Madhu Patil \\ Department of Electronics and Communication \\ Nitte Meenakshi Institute of Technology, Bangalore, \\ Karnataka, India
}

\begin{abstract}
Kroto Finder where 'Kroto' is a Greek word which means 'Crack', this in general gives an idea over the project where we use wireless communication in order to control and operate a robot which inspects the internal structure of a fluid pipe in order to determine any sorts of irregularities along with dents or other types of damages. Various types of sensors are mounted on the robot along with a surveillance camera with live streaming and recording capabilities. These sensors along with camera are connected to the robot and data which is derived from camera is transferred to the manually operated control station, by means of wireless communication. Wireless communication allows easy and uninterrupted transfer of data for continuous assessment. All sorts of decisions are taken by a manual supervisor based on the data which is received on the control station. Due to the high risk which is involved in the maintenance of these fluid pipes these decisions taken from time to time prove to be extremely useful and in turn can be used to avoid any sorts of accidents due to poor maintenance of these pipes which may result in damage to the Natural, Economic and Human resources. Therefore, the health of these pipes is of utmost importance and need to be regularly inspected internally and in a safe and feasible manner.
\end{abstract}

Keywords - Wireless Sensor Network (WSN), nrF24L01+, Arduino, Ultrasonic Sensor, ESP32-CAM Board.

\section{INTRODUCTION}

Pipes are the foremost vital mode of transportation for fluids, which include different types of fuels and other industrial products which are used actively in industries and other producing joints. A single day of inconvenience could cause an immense damage to various industries which in turn directly or indirectly affect the economy of the country. Therefore, industries along with government take immense care in making sure that all important fluids necessary are easily and quickly transported to the respective industries.

One among the leading problems encountered by organizations is the loss of fluids due to irregular functioning of pipes which not only have economical affects but also have immense effect on the environment. Among the various types of fluids which are transported through pipes some are combustible along with some of them being highly hazardous to nature which in turn may cause severe damage to the surroundings. Since, most of these transportation pipes are dug underground these damages cannot be immediately found out and continue to cause problems. There are no mechanisms as such which are capable of determining the exact position of damage or leaks which are happening in these pipes therefore when they have to be corrected the process becomes extremely time consuming since time is of utmost importance for the industries and processes involved in mending the pipes and making them useable once more becomes a vital process.

"PREVENTION IS BETTER THAN CURE" a universal saying that associates itself with all possible problems where it is better to avoid any problems instead of facing all involved 


\section{International Journal of Engineering Applied Sciences and Technology, 2020 Vol. 5, Issue 4, ISSN No. 2455-2143, Pages 505-514}

Published Online August 2020 in IJEAST (http://www.ijeast.com)

issues that arrives with it. Corrosion being an important factor in damaging the pipes, the pipes need to be checked for corrosion where in many cases these pipes are connected underground making surveillance almost impossible therefore creating a surveillance robot, which can travel underground in these pipes and determine the points or positions that are most likely to be affected of damage or leakage in immediate future and have to be immediately corrected.

Along with economic implications, we also have environmental damages which can be termed as deadly and most crucial problem if the leakage happens from these pipes. Some of the notable examples which can give the amount of damage caused are as following:

- On 17th June, 2017, a place in Jamnagar, Gujarat was flooded with oil after an oil pipe belonging to Indian Oil Corporation Limited ruptured causing leakage with initial reports suggested a pipe leakage due to poor maintenance which caused nearly 130 crores worth of loss to the existing agricultural land and IOCL was asked to pay immediate compensation to the defector and also shutting down industries in these areas creating both industrial and environmental damage.

- On 27th June, 2014, a gas pipeline exploded in Andhra Pradesh based industry killing 22 people. A report which was made on initial investigation provided enough evidence stating extensive pressure created at a section due to leakage which led to explosion of gas pipe. It also led to fire break out due to which many coconut trees, crops, cattle and wild birds were reduced to ashes.

- A GAIL pipeline leaked causing immense loss to property and agricultural land. Damages were caused due to extensive corrosion in pipe on 29th April, 2017. The reasons for this damage was found out to be corrosion and dent along with other physical damages occurring internally.

These few examples focus on telling that the gas pipelines which are said to be damaged may cause lot of damages to both life and property. Therefore, it is an important task where we have to inspect the pipe and monitor changes especially physical mechanical changes in order to avoid such accidents. Kroto finder is designed in such a way to continuously inspect the inner surroundings of the pipe which we consider as the important aspect in case of these pipes. We have two important modules whose involvement is to send and receive respective data from the inner pipe. This project is done on basis of study made on robotics where we continuously start to monitor the dents and damages in the pipe. The model which we have developed is an upgraded version compared to previous models as follows:

M. N. Mohammed et al. (2018) [1] describes the importance of the pipe inspection robots in industries and supply areas. In this paper they present a mobile autonomous robot which is able to reach the remote areas in the pipe. The paper also divides the robot design into three physical sections which helps in designing the robot in efficient way. The use of GSM module is used for connecting the device to a computer unit to get the exact location of the crack present in the robot. The camera present on the robot gives the real time coverage of the inner surface of the pipe which is used for effective detection, diagnosis of the crack.

Linija Shylin et al. (2017) [2] mainly presents the design that can be used to find cracks and leakage in the oil pipes by analyzing the change in the temperature and pressure inside the pipeline that cannot be detected by humans. This paper also gives a brief idea of usage of a wireless system to transfer the signals from the base controlling unit to various other controlling units operated under a single branch of operation. The usage of temperature and pressure sensor gives an advantage of using the robot in different diameters. The usage of Bluetooth gives the user to access the robot with the help of phone, making it as the base station.

V. Mallikarjuna et al. (2017) [3] propose a new type of design which uses three wheeled design which can adjust to the inner diameter of the pipe. In these types, robots can be varied as per the required diameter of the pipe to be tested. The kinematics of mechanism and actuator sizing of the robot also help in finding the crack as there will be variation if there are any irregularities. The usage of LED's gives a clear image of the pipes which makes the identification a lot easier.

Thanuja I K et al. (2016) [4] puts forward a robust design which is mainly helpful in detecting the cracks or irregularities that are present in gas pipes. The system continuously monitors the pressure and temperature in pipe as there will be variation if there is a crack or any debris of rust is present in pipes. The camera used in the system makes it helpful to check the amount of debris or the length of crack present in the pipes. The paper also shows the usage of ZigBee module for the data transmission between the control station and the robot.

This project is concerned about industrial applications of pipes, where in internal health of the pipe is inspected and necessary deformations which are unable to detect by human eye are rectified. In Mainline transmission pipes, the pipes involved are of different types which includes:

- Principle pipelines - Its diameter ranges from 16 to 48 inches

- Lateral pipelines - Its diameter ranges from 10 to 16 inches

- Interstate pipelines - Its diameter ranges from 24 to 36 inches

The pipe type which is popular and most extensively used are the Lateral Pipelines which are generally easy and comfortable to handle and major transportation of fluids are in and around these Lateral Pipes, therefore we have developed the robotic system which is easily operated inside the pipe. The Robot constructed need to be easily operated inside the pipes therefore we need to build it according to the dimensions of the pipe along with our feasibility to operate the instrument 


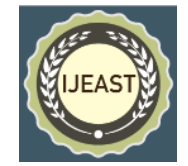

inside these pipes. Considering the dimensions of the lateral pipes, the robot dimensions are as follows:

Length -9 inches, Breadth -6 inches, Height -5.5 inches

The system is divided into two operations one is transmitting and other is receiving. It involves two operating stations one on the robot and the other one is the control station. These play an important role in operation where both the station behaves as transmitting and receiving centers, where these modules in general contribute to the effective working. Wireless medium used for controlling robot's movement is nRF24L01+ transceiver module and for transmitting live video data is Wi-Fi communication. High throughput of $\mathrm{Wi}-\mathrm{Fi}$ Communication enables seamless live video streaming of inner environment of the pipe.

\section{BLOCK DIAGRAM OF KROTO FINDER}

\section{A. Control Station -}

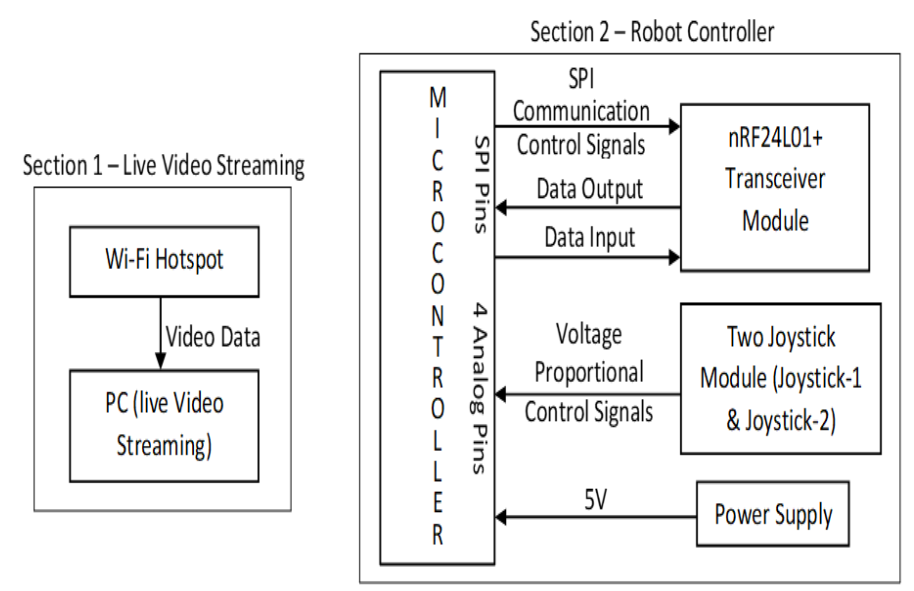

Fig. 1. Block Diagram of Control Station

The control station is an important part which controls the rotation of the motors of the robot thus controls the movement of the robot and also the camera cradle mounted on the robot which helps in the surveillance of the pipe with joystick 1 and joystick 2 respectively. Thus, control station behaves as a controlling module of the user. The information is transported to the robot using nRF24L01+ transceiver module along with this the live video data from the camera is also displayed to the technician on a camera web server. Camera uses Wi-Fi Communication medium to transmit live video data to control station.

\section{B. The Robot -}

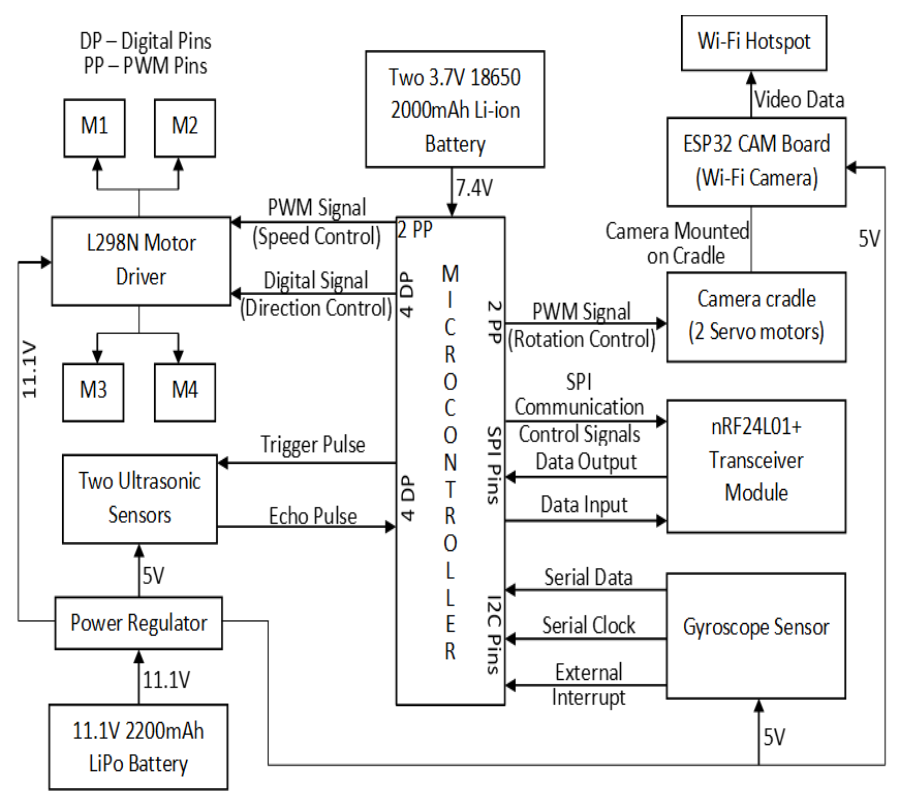

Fig. 2. Block Diagram of Robot

The robot side is the part that helps in surveillance of the pipe. It contains Arduino Mega Development Board containing Atmega2650 8-bit AVR microcontroller which is then interfaced along with sensors such as Ultrasonic Sensor (used to determine distance) and Gyroscope Sensor (used to balance the robot during its movement) where data is recorded by these sensors and sent to microcontroller. It has a L298N motor driver which controls the rotation of two motors. Our robot is a four wheeled robot where two motors on left side are shorted and operated as single motor. Similarly, two motors on right side are shorted and operated as single motor. Along with this we also have a 2-axis Camera Cradle which is used to hold the camera and rotate it in a desired direction. The movement of Camera cradle and DC motors are commanded upon receiving the commands from control station through nRF24L01+ Transceiver Module. It has an ESP32 CAM Board which is embedded with OV2640 Camera to record the video inside the pipe and then transmit it through inbuilt Wi-Fi to be displayed on the laptop or necessary device. Power is supplied to the system using a 11.1V Lithium Polymer Battery and Two 3.7V Li-ion batteries are used for Arduino because Servo motors are high current driven devices and current produced by $11.1 \mathrm{~V}$ LiPo battery is $3 \mathrm{~A}$ and maximum of the power will be consumed by DC motors thus leading to supply of insufficient power to servo motors. Power supply from LiPo battery is regulated to $5 \mathrm{v}$ by a step-down buck converter and supplied to various modules mounted on robot. 


\section{IMPLEMENTATION BY SENSORS}

\section{A. Ultrasonic Sensor -}

HC-SR04 is the ultrasonic sensor most widely used for robotics applications where using SONAR technology we are able to determine the distance between any objects and the moving robot which in turn helps in avoiding collision and allows robot to escape such unexpected situations.

HC-SR04 sensor allows a non-contact method of operation. It has an ultrasonic trigger along with a control circuit and an echo. Ultrasonic waves are emitted from the trigger onto any solid object/barrier and then the ultrasonic waves are received back by the echo. Trigger is set high for $10 \mu$ s. This will send 8 cycle sonic burst at $40 \mathrm{kHz}$. Time plays an important role in this operation where a control circuit determines the emitted time to received time in microseconds and using simple mathematical calculation distance is determined between the robot and the solid object.

The following Equation constitute the mathematical calculation of the distance between the object and the Ultrasonic Sensor:

$$
\mathrm{s}=\mathrm{v} * \mathrm{t}
$$

Where ' $\mathrm{s}$ ' is the distance between the object and the robot, ' $\mathrm{v}$ ' is the speed of a sound wave i.e. $340 \mathrm{~m} / \mathrm{s}$ and ' $\mathrm{t}$ ' is defined as time taken for an ultrasonic wave to travel from an emitter to a receiver. The speed of sound is converted into $\mathrm{cm} / \mu$ s i.e. $0.034 \mathrm{~cm} / \mu \mathrm{s}$. the modified equation is:

$$
\mathrm{s}=0.034 * \mathrm{t}
$$

The obtained distance value consists of distance it takes to hit the object and come back. Hence the obtained value is divided by 2 to eliminate the distance taken by wave to comeback after hitting the object. So, the final equation will be:

$$
\mathrm{s}=(0.034 * \mathrm{t}) / 2
$$

\section{B. Gyroscope Sensor -}

A 3-axis Gyroscope is combined with a 3-axis Accelerometer on a single Silicon Chip which together is termed has a MPU6050 sensor. It is even capable of solving crossalignment problems that is an issue in discrete parts along with 9 axis motion Fusion algorithms which can be solved using these sensors. The gyroscope sensor used is essential to determine the balance of the robot inside the pipe. This sensor is responsible for uninterrupted movement of the robot inside the pipe which safeguards the robot from tripping over or unbalancing causing any sort of obstruction inside the pipe. It is used to recognize the angular velocity considering $\mathrm{X}, \mathrm{Y}$ and $\mathrm{Z}$ axes.

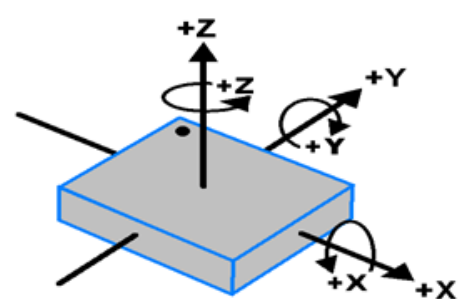

Fig. 3. MPU6050 Orientation and Polarity of Rotation

When the Gyroscope Sensor is displaced along the sense axes, the Coriolis Effect takes place which produces a vibration that will be sensed by MEMS System placed inside the Sensor. Amplification, Demodulation, Filtering and Digitization processes are performed on this signal. This sensor measures the angular velocity along each axis in DPS.

These obtained angular velocities are then normalized and used to calculate Pitch, Roll and Yaw along Y, X and $\mathrm{Z}$ axes respectively. The following equation depict the calculation of Roll, Pitch and Yaw:

$$
\begin{gathered}
\text { Roll }=\text { Roll }+ \text { normalized X-Axis } * \text { timeStep } \\
\text { Pitch }=\text { Pitch }+ \text { normalized } \text { Y-Axis } * \text { timeStep } \\
\text { Yaw }=\text { Yaw + normalized Z-Axis } * \text { timeStep }
\end{gathered}
$$

\section{FLOWCHART REPRESENTATION}

\section{A. Flowchart of Robot -}

The Flowchart of the robot represents the working of the robot from the development point of view, its various commands and operations are aimed at fulfilling the respective objective of the project. From the start or after power ON immediately the robot establishes communication with the control station in order to receive important control commands from the control station using radio.read() command. The Camera becomes the first element that starts recording video of inner surface of the pipe. Immediately the ultrasonic sensor emits ultrasonic waves for $10 \mu \mathrm{s}$, this achieved by commanding ultrasonic sensor's trigger pin using command digitalWrite(). Once these waves hit the obstacle and are received by ultrasonic sensor, the sensor calculates the time of travel of these waves and sends it to microcontroller. Then microcontroller calculates the distance from the robot to the object using the formula mentioned in Eq.(3). Now, an IF statement comes into action where if the threshold distance is greater than distance, if Yes, the angle of alignment is checked using MPU6050 gyroscope sensor or else the robot stops its movement. Once the angle is checked for alignment, in case of measured angle greater than threshold, the backward movement is allowed and if the distance is less, the joystick can command the motor for its movement. 
International Journal of Engineering Applied Sciences and Technology, 2020

Vol. 5, Issue 4, ISSN No. 2455-2143, Pages 505-514

Published Online August 2020 in IJEAST (http://www.ijeast.com)

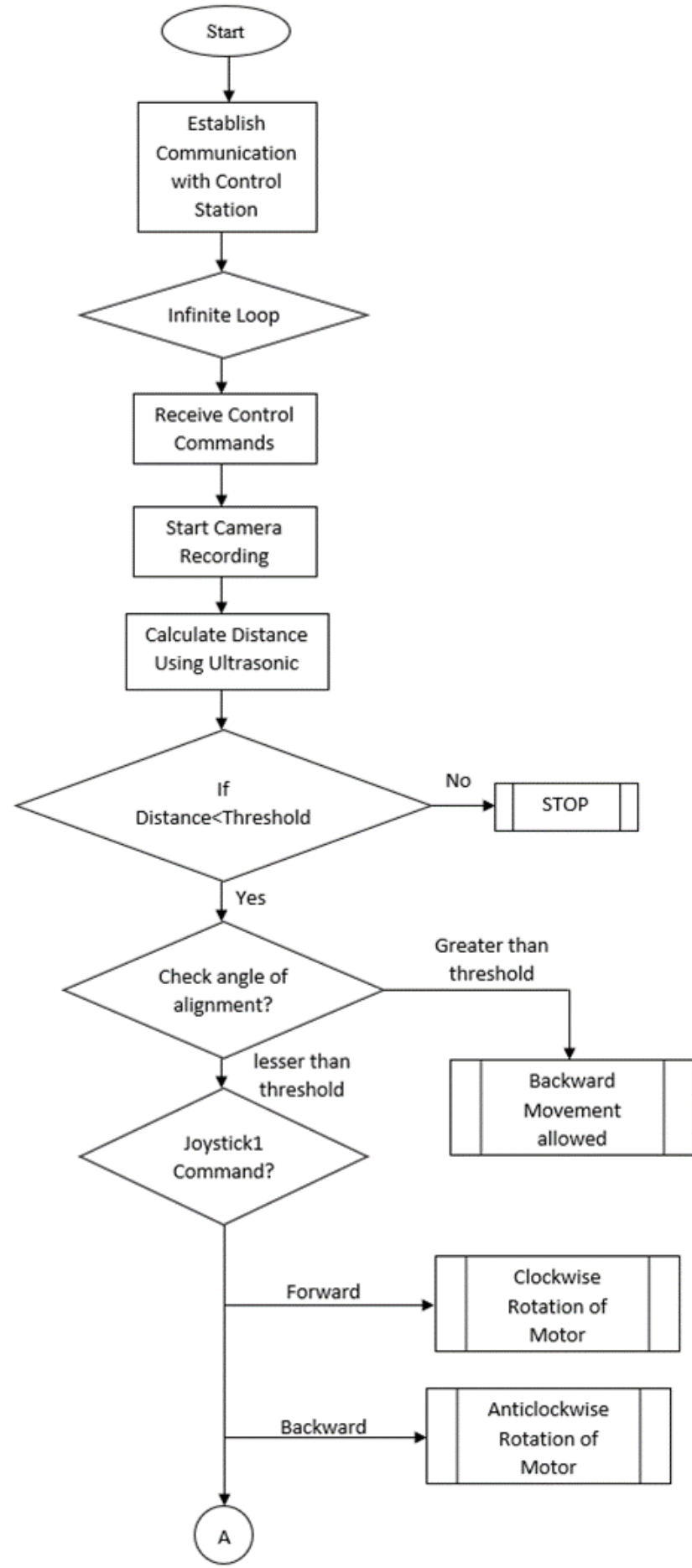

Fig. 4. Flowchart of Robot: Part 1

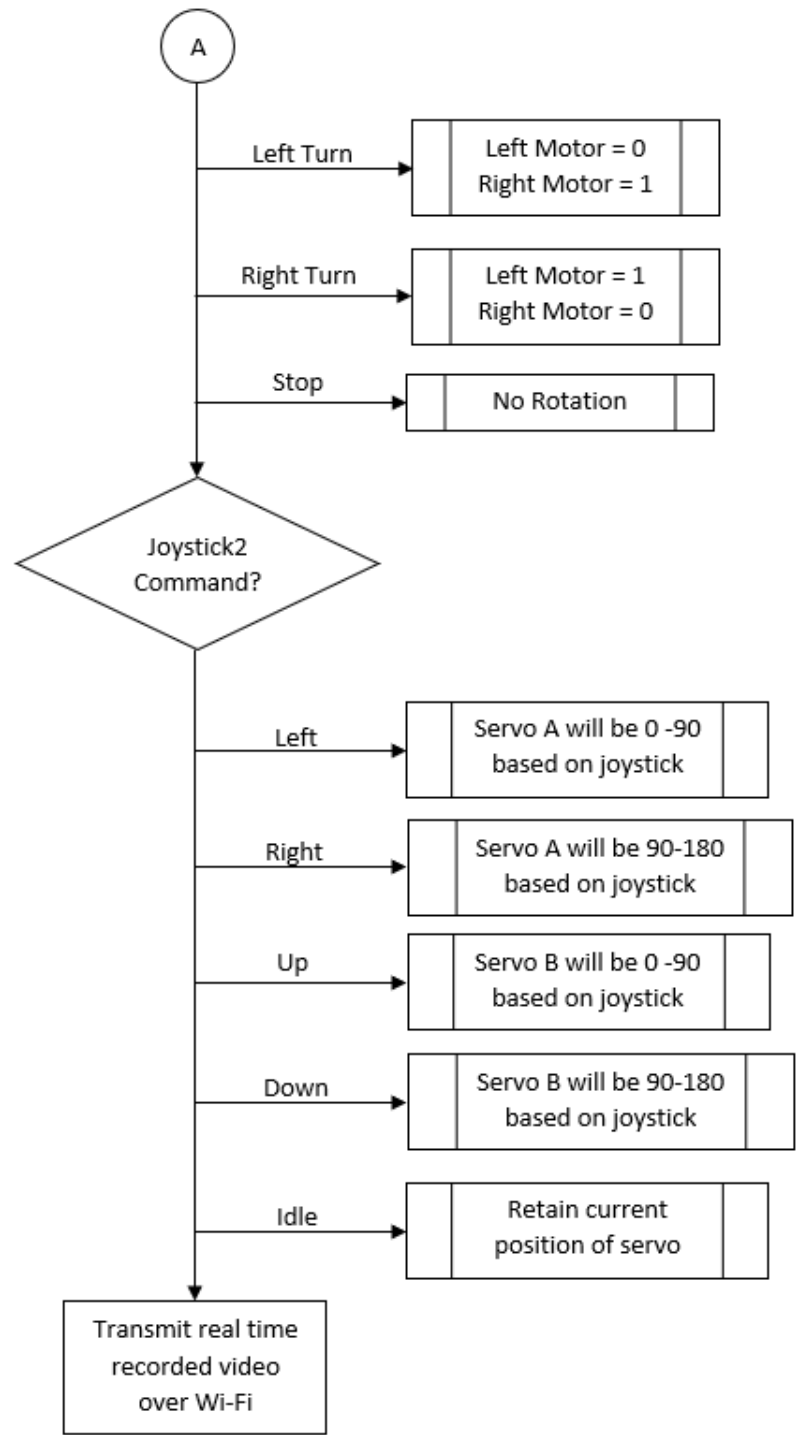

Fig. 5. Flowchart of Robot: Part 2

\section{B. Flowchart of Control Station -}

The Control Station follows a simple method of operation, at power ON the system connects or starts communicating with the robot side through nRF24101+ device then immediately taking analog signals from joystick as input to the converter system to convert it to digital signal (specifically into values ranging from $0-1023$ ) and then receiving the live video over Wi-Fi and displaying it on the PC's Camera Web Server for proper surveillance. Using this live streaming, the operator will generate a report regarding the overall health of the pipes. If health of the pipe section is poor then that particular section of pipe will be dismantled and will be repaired or replaced. 


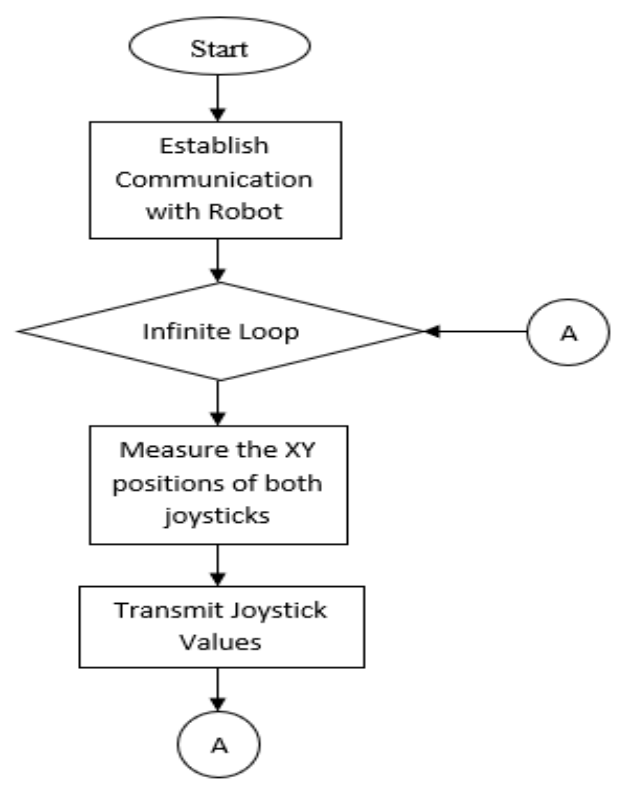

Fig. 6. Flowchart of Control Station

\section{RESULTS AND DISCUSSION}

Simulation and programming were accompanied using a system which had 8GB of RAM and 64-bit Windows 10 OS. We have used Proteus 8 simulation software to simulate electronic designs and Arduino software for programming Arduino board. We have conducted simulation study on Ultrasonic Sensor and LM298N Motor Driver to verify programming logic before being tested directly on hardware.

\section{A. Simulation of L298N Motor Driver -}

Simulation of L298N Motor Driver was performed to review the functioning of DC motors. Speed control of DC motor is consistently done by Pulse Width Modulation (PWM).

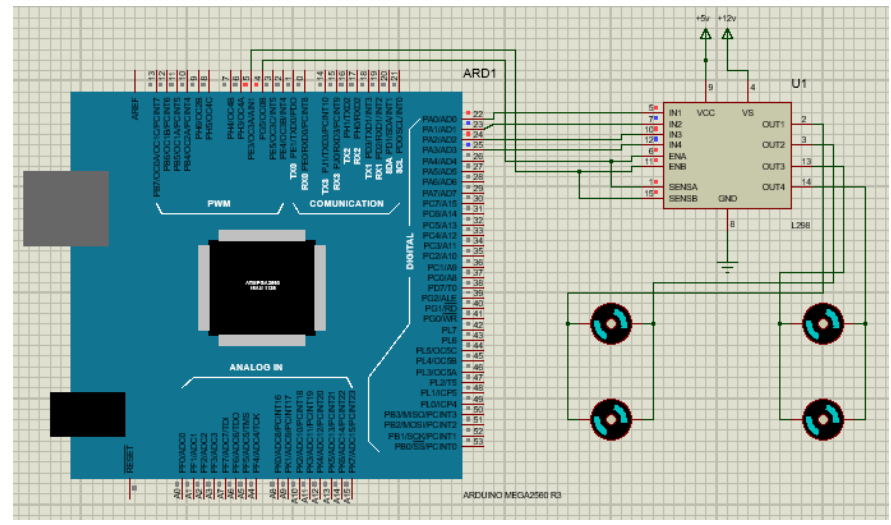

Fig. 7. Schematic Layout of Speed Control of DC Motor with L298N Motor Driver
Generation of PWM are often achieved by using Arduino. This pulse is then given to L298N Enable Pin to manage the motor speed. Speed of a DC motor is regulated by altering the duty cycle of PWM signal. This is often achieved by simple Arduino programming. For instance, analogWrite(Enable, 255 ) corresponds to $100 \%$ of duty cycle i.e. motor rotates at full speed and analogWrite(Enable, 0) corresponds to $0 \%$ of duty cycle i.e. motor won't rotate. Direction of rotation of motor is controlled by Arduino commands digitalWrite(high) or digitalWrite(low) using two input pins of L298N to a motor. Arduino control commands for different duty cycle values is show in the table 1 .

Table -1 Speed Controlling of DC Motor Using Arduino

\begin{tabular}{|c|c|c|c|}
\hline \multicolumn{4}{|c|}{ Speed Controlling of DC motors Using Arduino } \\
\hline $\begin{array}{c}\text { Sl. } \\
\text { No. }\end{array}$ & $\begin{array}{c}\text { Duty Cycle } \\
(\%)\end{array}$ & Command & Motor Status \\
\hline 1 & 0 & analogWrite(0) & No Rotation \\
\hline 2 & 19.6 & analogWrite(50) & Rotating \\
\hline 3 & 25 & analogWrite(64) & Rotating \\
\hline 4 & 50 & analogWrite(127) & Rotating \\
\hline 5 & 75 & analogWrite(191) & Rotating \\
\hline 6 & 100 & analogWrite(255) & Rotating \\
\hline
\end{tabular}

L298N Motor Driver has a capacity of controlling two DC motors at a time. In our project, we have developed a four wheeled robot in which two motors on the left side of the chassis are shorted and connected as one motor to L298N Motor Driver and other two motors on right side are shorted and connected as one motor to L298N Motor Driver. This motor driver has four motor direction control pins of which IN1 and IN2 are associated with left motor and IN3 and IN4 are associated with right motor. Below table shows the direction control of motors using Arduino:

Table - 2 Direction Controlling of DC Motor Using Arduino

\begin{tabular}{|c|c|c|c|c|c|}
\hline \multicolumn{5}{|c|}{ Direction Controlling of DC Motor using Arduino } \\
\hline $\begin{array}{c}\text { Sl. } \\
\text { No. }\end{array}$ & IN1 & IN2 & IN3 & IN4 & Function \\
\hline 1 & High & Low & High & Low & Forward Movement \\
\hline 2 & Low & High & Low & High & Backward movement \\
\hline 3 & High & Low & Low & Low & Forward Right Turn \\
\hline 4 & Low & High & Low & Low & Backward Right Turn \\
\hline 5 & Low & Low & High & Low & Forward Left Turn \\
\hline 6 & Low & Low & Low & High & Backward Left Turn \\
\hline
\end{tabular}

\section{B. Simulation of Ultrasonic Sensor -}

Figure 8 shows the schematic layout of interfacing Ultrasonic Sensor with Arduino Mega Board. In practical scenario, we send ultrasonic waves to determine distance between 


\section{International Journal of Engineering Applied Sciences and Technology, 2020 Vol. 5, Issue 4, ISSN No. 2455-2143, Pages 505-514 \\ Published Online August 2020 in IJEAST (http://www.ijeast.com)}

ultrasonic sensor and obstacle whereas in case of simulation we used a $10 \mathrm{k} \Omega$ potentiometer using which we can vary voltage to record different distances. Distance values are displayed on virtual monitor of proteus software.

The results of both the simulation shows that the program used is logically valid and it can be used for hardware testing. Hardware testing is done to check the working of the hardware and expected output before assembling on the robot. Hardware testing of L298N motor driver was performed and we obtained expected results which were similar to simulation results.

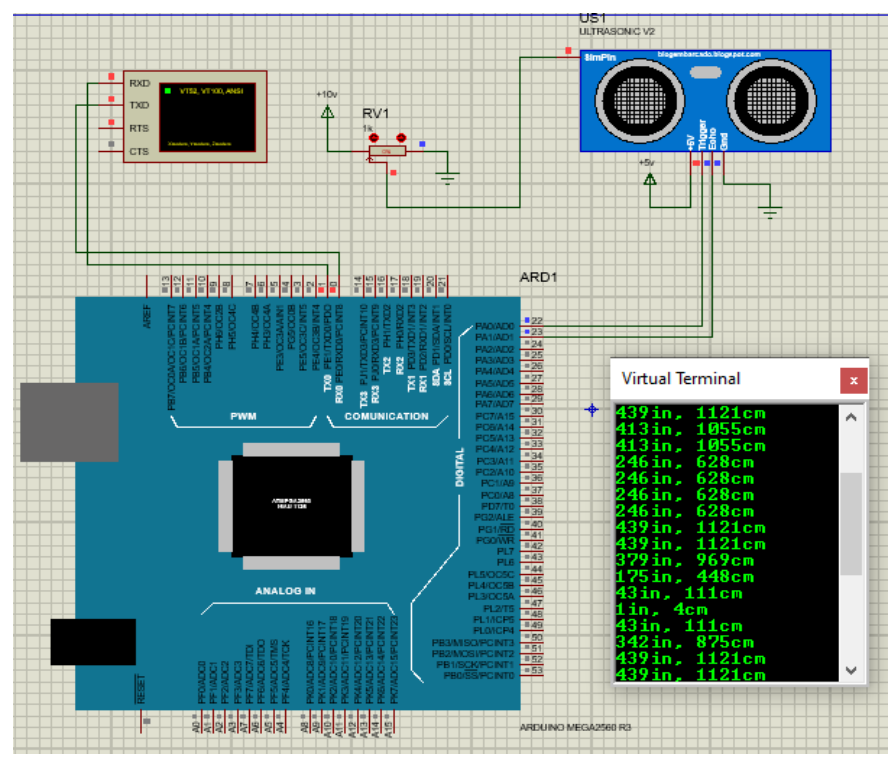

Fig. 8. Schematic Layout of Interfacing Ultrasonic Sensor with Arduino

\section{Hardware Testing of Ultrasonic Sensor -}

Hardware testing of ultrasonic sensor was experimented by measuring the distance between obstacle and the robot. The results for various testcases were monitored in Arduino's serial monitor. The experimental results are tabulated as follows:

Table -3 Testcases Of Ultrasonic Module Testing

\begin{tabular}{|c|c|c|c|}
\hline \multicolumn{4}{|c|}{ Ultrasonic Module Distance Check } \\
\hline $\begin{array}{c}\text { Sl. } \\
\text { No. }\end{array}$ & $\begin{array}{c}\text { Actual } \\
\text { Distance }(\mathrm{cm})\end{array}$ & $\begin{array}{c}\text { Measured } \\
\text { Distance }(\mathrm{cm})\end{array}$ & Error $(\mathrm{cm})$ \\
\hline 1 & 4 & 4.05 & 0.05 \\
\hline 2 & 7 & 7.08 & 0.08 \\
\hline 3 & 15 & 15.15 & 0.15 \\
\hline 4 & 25 & 24.80 & 0.20 \\
\hline 5 & 50 & 49.71 & 0.29 \\
\hline 6 & 75 & 75.10 & 0.10 \\
\hline 7 & 100 & 99.5 & 0.50 \\
\hline
\end{tabular}

The experimental results show that the measured distance is approximately equal to the actual distance between the sensor and the object with an error not exceeding $0.5 \mathrm{~cm}$ which is observed when the distance between the obstacle and the robot is large. Figure 9 and 10 depicts the graphical representation of experimental results of ultrasonic sensor testing:

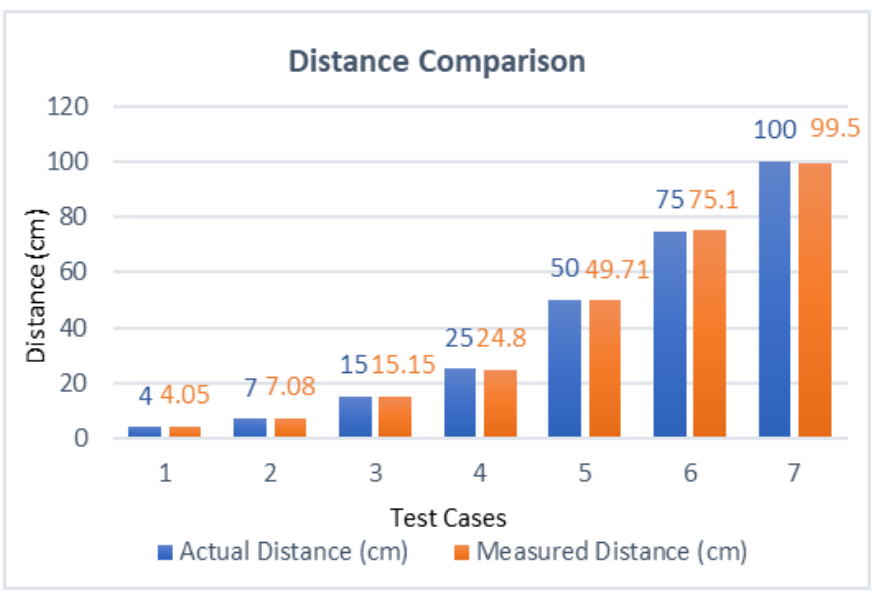

Fig. 9. Distance Comparison Between Actual Distance and Measured Distance

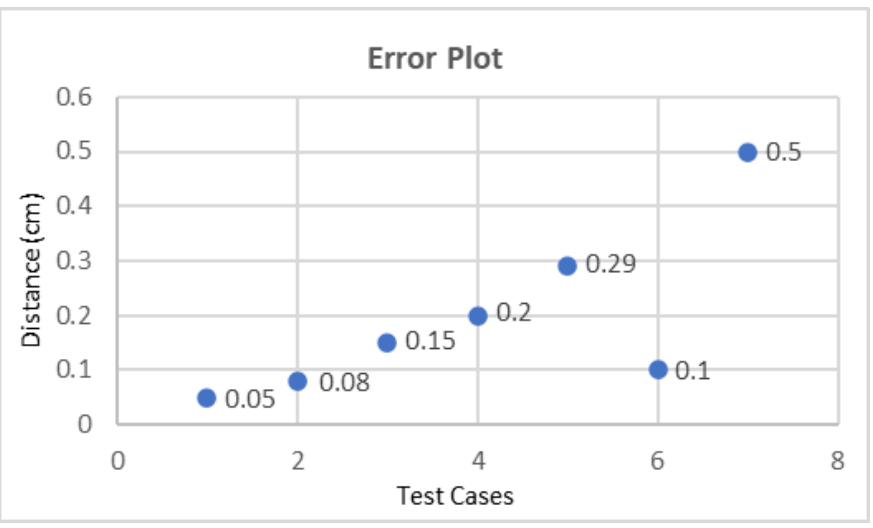

Fig. 10. Error Points of Ultrasonic Sensor

\section{Hardware Testing of MPU6050 Gyroscope Sensor -}

Hardware testing of MPU6050 gyroscope sensor was performed to determine the angle accuracy measured by the sensor. After uploading the program related to testing of gyroscope sensor to Arduino Mega, it connects MPU6050 to Arduino Mega through I2C bus interface. We used a reference plane which had markings of all angle values and the MPU6050 gyroscope sensor was rotated according to the markings on reference plane. this sensor produces output which includes angular velocities along $\mathrm{X}, \mathrm{Y}$ and $\mathrm{Z}$ axes. Based on the positioning of the sensor on robot chassis, roll angle was of much importance which is measured along $\mathrm{X}$ axis and will be helpful in determining in alignment of the robot inside the pipe. If this roll value exceeds the threshold 


\section{International Journal of Engineering Applied Sciences and Technology, 2020 Vol. 5, Issue 4, ISSN No. 2455-2143, Pages 505-514 \\ Published Online August 2020 in IJEAST (http://www.ijeast.com)}

value then we can stop the robot from moving further. The experimental results show that the measured angle is approximately equal to actual angle with an error angle not exceeding $\pm 0.66^{\circ}$. This testing was performed for various testcases and the results are tabulated and figure 11 and 12 depicts the graphical representation of experimental results of MPU6050 gyroscope sensor testing.

Table - 4 Testcases Of MPU6050 Gyroscope Sensor Testing

\begin{tabular}{|c|c|c|c|}
\hline \multicolumn{4}{|c|}{ Roll Angle Measurement using MPU6050 Gyroscope } \\
Sensor \\
\hline $\begin{array}{c}\text { Sl. } \\
\text { No. }\end{array}$ & $\begin{array}{c}\text { Actual Angle } \\
(\mathrm{deg})\end{array}$ & $\begin{array}{c}\text { Measured } \\
\text { Angle (deg) }\end{array}$ & Error (deg) \\
\hline 1 & 0 & 0 & 0 \\
\hline 2 & 10 & 9.88 & -0.12 \\
\hline 3 & 20 & 20.64 & 0.64 \\
\hline 4 & 30 & 30.01 & 0.01 \\
\hline 5 & 40 & 40.44 & 0.44 \\
\hline 6 & 45 & 45.5 & 0.50 \\
\hline 7 & 50 & 49.34 & -0.66 \\
\hline 8 & 60 & 59.39 & -0.61 \\
\hline 9 & 70 & 70.19 & 0.19 \\
\hline 10 & 80 & 80.01 & 0.01 \\
\hline 11 & 90 & 89.56 & -0.44 \\
\hline
\end{tabular}

Figure 11 and 12 depicts the graphical representation of experimental results of gyroscope sensor testing:

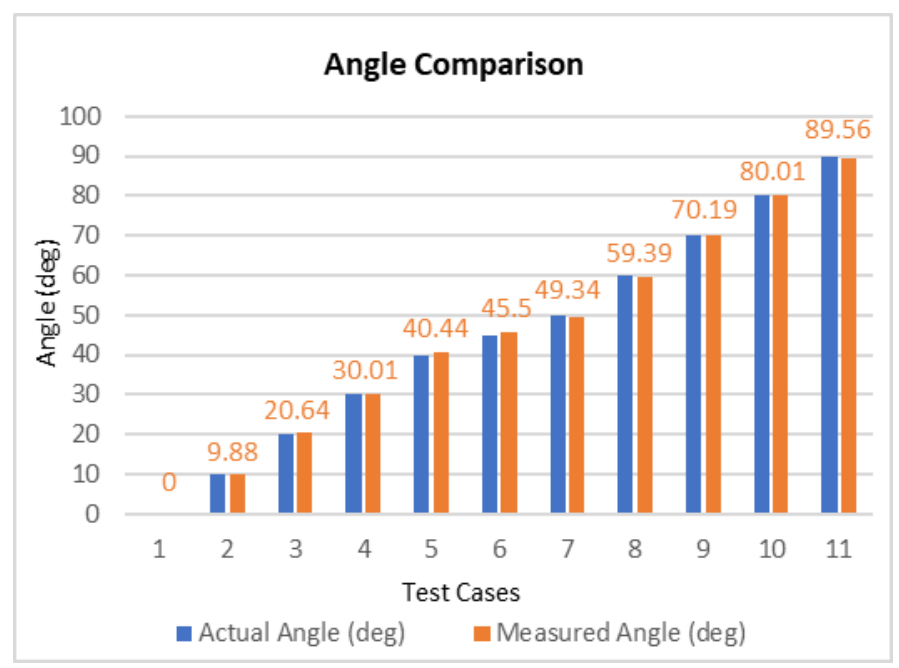

Fig. 11. Angle Comparison Between Actual Angle and Measured Angle

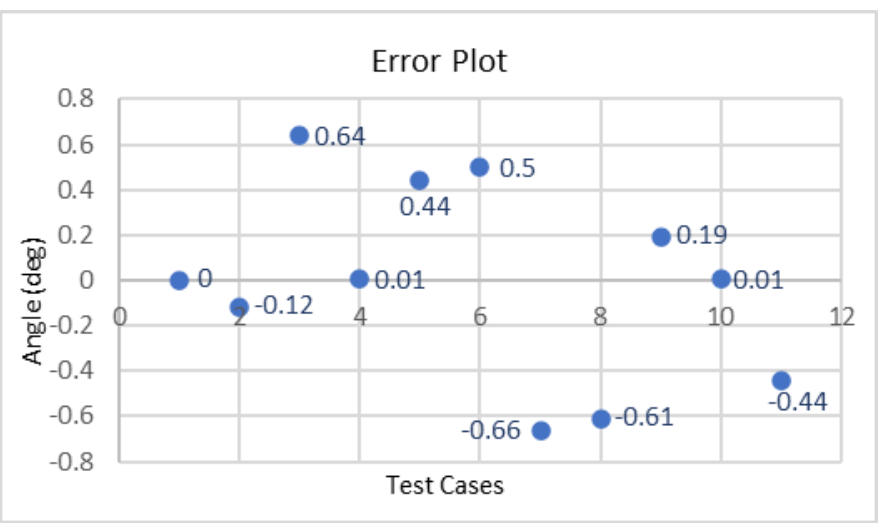

Fig. 12. Error Points of MPU6050 Gyroscope Sensor

\section{E. Overall Results -}

The proposed system was implemented, developed and desired results were obtained. The robot was tested on ground to check the working. The control station successfully transmitted control commands from both the joystick through nRF24L01+ transceiver module. The robot upon receiving the control commands, desired actions were taken by microcontroller. Microcontroller was continuously monitoring the distance and angle values produced by Ultrasonic and Gyroscope Sensor and if there were any deviation in values then desired action was taken by controller. ESP32 CAM Board successfully recorded the video and live streaming was done at the control station end through a Camera Web Server. Below are few pictures of fully developed robot and control station.

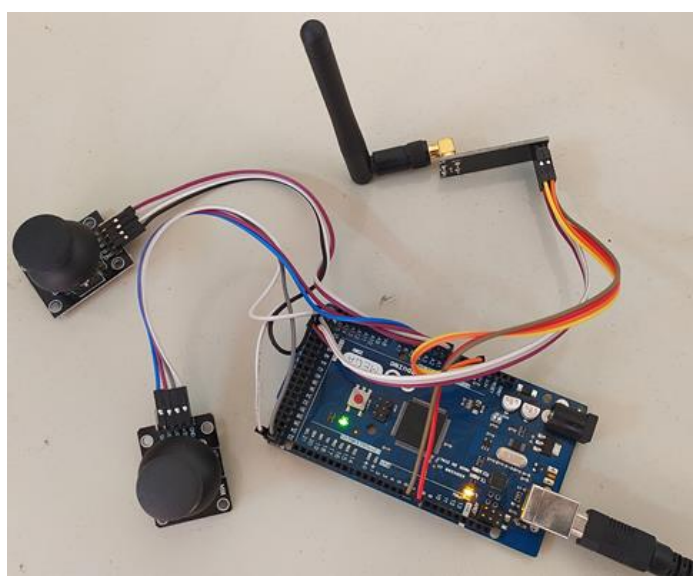

Fig. 13. Control Station End 


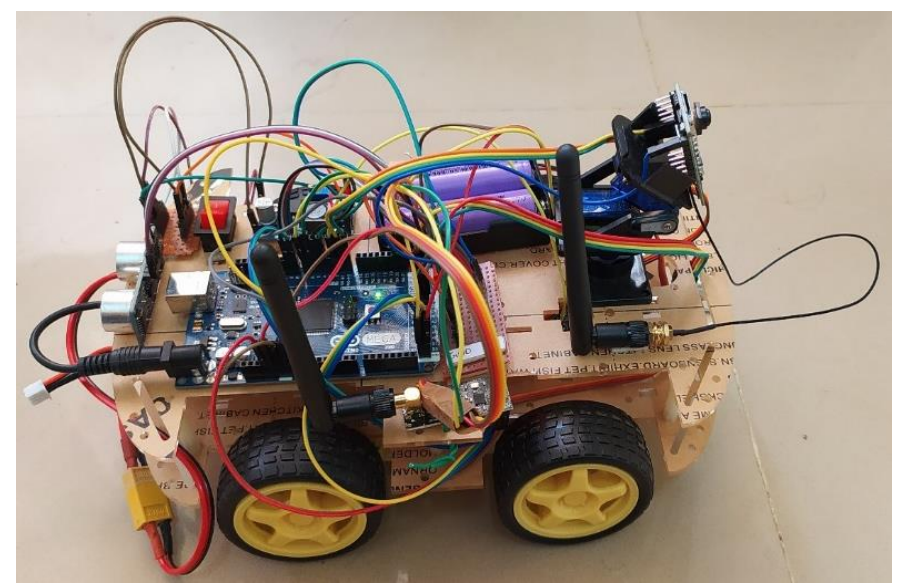

Fig. 14. Side View of Robot

\section{CONCLUSION}

The process of maintaining fluid pipes is a very crucial and important process for which we consider a better system of manual surveillance, where we use live and also recorded data from the robot inside the transportation pipe to determine the quality of pipe which is used. If any sort of deviation or damages is observed by the technician the pipe is asked to be replaced or repaired. The wireless mode of communication along with low risk use of electronic surveillance equipment allows the faster and safer mode or medium of inspection in the system. With the use for modern technology we can surely be able to design an immensely effective method of operation from which we can devise a better system of inspection.

\section{ACKNOWLEDGEMENT}

The satisfaction and euphoria that accompanies the successful completion of any project would be incomplete without the mention of people who made it possible and whose constant encouragement and guidance has been a source of inspiration throughout the work.

We would like to express our deepest sense of gratitude for our beloved Director Prof. N R Shetty for providing us a great learning environment. We humbly extend our immense gratitude for our beloved Principal Dr. H.C. Nagaraj for continuous support and encouragement. We cannot thank enough our dear Head of the Department Dr. Ramachandra A. $\mathrm{C}$ for the continuous efforts in creating a competitive environment in the department and encouragement throughout the course of completion of this project.

With profound gratitude, we express our indebtedness to our guide Dr. Madhu Patil, whose dedication to the project, knowledge and references got our job done in the shortest time frame possible. Madam, we would like to sincerely thank you for the quality time you spent with us to clarify our doubts and for guiding us at each and every step towards completion of this project.

We are also thankful to the entire Department of Electronics and Communication engineering for the co-operation and suggestions without which this project would not have been a successful one.

We also thank all our friends who directly or indirectly helped us a lot in this project completion.

\section{REFERENCES}

[1] M. N. Mohammed, Vidya Shini Nadarajah, Nor Fazlina Mohd Lazim (2018), "Design and development of Pipeline inspection Robot for Crack and Corrosion Detection", Proceedings of IEEE Conference on Systems, Process and Control (ICSPC 2018), Melaka, Malaysia, December 2018, (pp. 29-32)

[2] Linija Shylin, Sudha M, Prasoona O, Rhituporna Sarkar, Tejashree M S (2017), "Development of Android Based Remote Acquisition - Kroto finder", IJERECE 2017, Vol. 4, Issue 5, (pp. 148-153)

[3] V. Mallikarjuna, K. Rajesh, C. Chandra Sekhara (2017), "Design and Development of Kroto finder", Department of ME, Joginapally BR Engineering College, Hyderabad, India.

[4] Thanuja I K, Sheeba Kumari M, Vineeth Reddy M (2016), "Kroto finder- detection of damages in oil/gas pipes”, IJAER 2016, Vol. 12, Issue VI, (pp. 19-30)

[5] Shahid Latif, Syed Adil Javed, Zil e Huma, Izzat Fatima, Arsalan Ejaz, Umair Naeem (2019), "Design and development of a wireless controlled human aide robot", Proceedings of IEEE International Symposium on Recent Advances in Electrical Engineering (RAEE 2019), Vol. 4, 2019

[6] Lang Chen, Jianbo Zhang, Yao Wang (2018), "Wireless car control system based on Arduino UNO R3", Proceedings of IEEE 2nd Advanced Information Management, Communicates, Electronic and Automation Control Conference (IMCEC 2018), (pp. 1783-1787)

[7] Bolun Zeng, Lang Chen, Jianbo Zhang, Yao Wang (2018), "Self Balancing Car Based on Arduino Uno R3", Proceedings of IEEE 3rd Advanced Information Technology, Electronic and Automation Control Conference (IAEAC 2018), (pp. 1939-1943)

[8] Anggi Sahfutri, Nyayu Latifah Husni, M. Nawawi, Iskandar Lutfi, Evelina, Ade Silvia, Ekawati Prihatini (2018), "Smart Parking Using Wireless Sensor Network System", Proceedings of IEEE International Conference on Electrical Engineering and Computer Science (ICECOS 2018), (pp. 117-122)

[9] Pertab Rai, Murk Rehman (2019), "ESP32 Based Smart Surveillance System", Proceedings of IEEE International 


\section{International Journal of Engineering Applied Sciences and Technology, 2020 \\ Vol. 5, Issue 4, ISSN No. 2455-2143, Pages 505-514 \\ Published Online August 2020 in IJEAST (http://www.ijeast.com)}

Conference on Computing, Mathematics and Engineering Technologies - iCoMET 2019

[10] Lubna Najjar, Noor Johari, Manar Qamhieh (2019), “A Leader-Follower Communication Protocol for MultiAgent Robotic Systems", Proceedings of IEEE Jordan International Joint Conference on Electrical Engineering and Information Technology (JEEIT 2019), (pp. 742-747)

[11] Aashvij Shenai, Abhijeet Antin, Pallvai R, Dawnee Soman (2018), "Development of In-Pipe Robot for Assisting Borewell Rescue Operations", Proceedings of IEEE 3rd International Conference on Circuits, Control, Communication and Computing (I4C) 2018

[12] Faris Alshehri, A. H. M. Almawgani, Ayed Alqahtani, Abdurahman Alqahtani (2019), "Smart Parking System for Monitoring Cars and Wrong Parking", Proceedings of IEEE 2nd International Conference on Computer Applications \& Information Security (ICCAIS 2019), (pp. 1-6)

[13] Miguel Molina, Angelo Vera, Carolina Molina, Priscila Garzon (2018), "Design and Construction of an Obstacle Avoiding Robot Based on Arduino Platform and Programming Tools", Proceedings of 9th IEEE Annual Ubiquitous Computing, Electronics \& Mobile Communication Conference (UEMCON 2018), (pp. 788791)

[14] Ahmad Bazli Bakri, Ramli Adnan, Fazlina Ahmat Ruslan (2019), "Wireless Hand Gesture Controlled Robotic Arm Via NRF24L01 Transceiver", Proceedings of IEEE 9th Symposium on Computer Applications \& Industrial Electronics (ISCAIE 2019), (pp. 16-22)

[15] Stefan Kljakic, Vladimir Rajs, Milan Bodic, Nemanja Cvetkovic (2019), "Position Regulation System with Camera and Microcontroller ESP32", Proceedings of IEEE EUROCON - 18th International Conference on Smart Technologies (ICST 2019), (pp. 1-6)

[16] Antonio Carlos Bento, Leonardo Augusto Taniguti Mantovani, Norberto Santos (2019), "A Data Transmission Survey with NodeMCU12e with Motor Shield and Nextion + NRF24L01", Proceedings of IEEE 10th International Conference on Computing, Communication and Networking Technologies (ICCCNT) 2019

[17] Vinod Kumar Singh, Abhishek Sahu, Atahar Beg, Bushra Khan (2018), "Speed \& Direction Control of DC Motor through Bluetooth HC-05 Using Arduino", Proceedings of IEEE 2018 International Conference on Advanced Computation and Telecommunication (ICACAT) 2018

[18] Prem Prakash Murmu, Dr. Roopa Jeyasingh J, Alexander Joe Timothy (2019), "A Novel modernistic techniques in women security system using ESP32 and Arduino Uno", Proceedings of IEEE 2nd International Conference on Signal Processing and Communication (ICSPC 2019), Coimbatore, India, (pp. 330-334)
[19] Nazir Ahmmed, Nusrat Jahan Jenny, Anika Ibnat Binte Alam, Md. Adnan Arefeen (2019), "VADet: An Arduino based automated vehicle accident detection and messaging system", Proceedings of IEEE 1st International Conference on Advances in Science, Engineering and Robotics Technology (ICASERT) 2019

[20] Oleh Horyachyy (2017), "comparison of wireless communication technologies used in smart home", Blekinge Tekniska Hogskola 2017 